\title{
Dietary Polyphenols, Deacetylases and Chromatin Remodeling in Inflammation
}

\author{
Irfan Rahman Sangwoon Chung \\ Department of Environmental Medicine, Lung Biology and Disease Program, University of Rochester \\ Medical Center, Rochester, N.Y., USA
}

The therapeutic benefits of fruits, vegetables, tea and wine are mostly attributed to the presence of phenolic compounds. Naturally occurring dietary polyphenols, such as curcumin (diferuloylmethane, an active component of spice turmeric) and resveratrol (phytoalexin, a flavanoid found in red wine) can modulate signaling pathways mediated via NF- $\mathrm{kB}$ and MAP kinase, and up-regulate glutathione biosynthesis genes through activation of Nrf2. Polyphenols also down-regulate the expression of pro-inflammatory mediators, matrix metalloproteinases and adhesion molecules by inhibiting histone acetyltransferase (HAT) activity and activating histone deacetylases (HDACs)/sirtuins. It has been reported that in severe asthma and in chronic obstructive pulmonary disease (COPD) patients, oxidative stress not only activates the NF- $\mathrm{kB}$ pathway but also alters the histone acetylation and deacetylation balance via post-translational modifications of HDACs. Corticosteroids have been one the major modes of therapy against various respiratory diseases, such as asthma and COPD. Failure of corticosteroids to ameliorate such disease conditions is due to the reduction of HDAC2 and SIRT1 levels/activities in lungs of asthmatics and COPD patients. Dietary polyphenols, such as curcumin, resveratrol, and catechins have been reported to modulate epigenetic alterations in various experimental models. The anti-inflammatory property of curcumin, resveratrol, and catechins is associated with their ability to induce HDAC activity and thereby restore the efficacy of glucocorticoids or overcome its resistance. Thus, these polyphenolic compounds have therapeutic value as antioxidants, anti-inflammatory therapy and adjuvant therapy with steroids against chronic inflammatory and epigenetically-regulated diseases. In this chapter we present the current knowledge on the mode of action of these polyphenols in the light of HDACs. 
Fig. 1. Regulation of epigenetic events by polyphenols. Dietary polyphenols inhibit epigenetic modifications, such as DNA methylation and histone modification through regulation of DNA methyltransferase, CBP/HAT activity, and deacetylases leading to resolution of inflammation. Thus, this is a possible way forward to design therapeutic strategies for human lung diseases by epigenetic modifications.

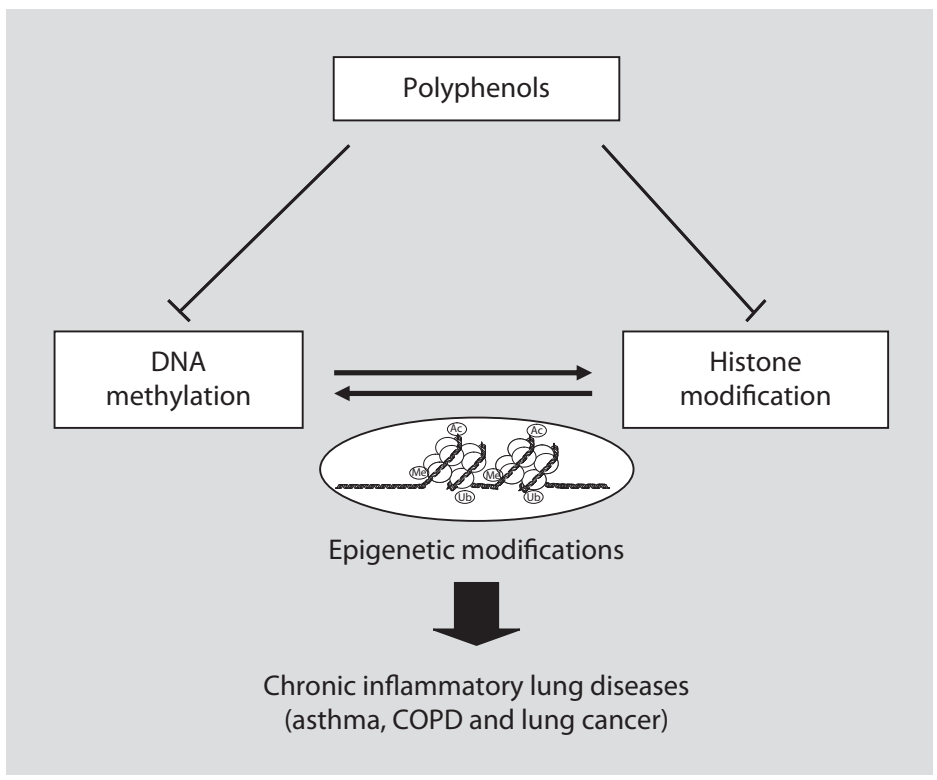

\section{Polyphenols: An Overview}

A variety of dietary plants contain polyphenols which impart disease prevention abilities to fruits and vegetables. Polyphenols are secondary metabolites of plants and represent a vast group of compounds having aromatic ring(s), characterized by presence of one or more hydroxyl groups with varying structural complexities. The most widely distributed group of plant phenolics are flavonoids. The flavonoids subclasses comprise of flavonols, flavones, flavanols, isoflavones, antocyanidins, and others. In this chapter, we will consider the biological properties, with special reference to epigenetics (histone acetylation/deacetylation) and inflammation, of some well-known and well-studied polyphenols, such as resveratrol, curcumin, and catechins.

\section{Modulation of Inflammation by Polyphenols}

Investigations into the mechanism of action of polyphenols have revealed that polyphenols may modulate cellular signaling during inflammation [1-3]. In the following sections, some individual polyphenolic compounds will be discussed, especially their anti-inflammatory properties which impart their effects via chromatin/epigeneticsdeacetylase modifications (fig. 1).

\section{Resveratrol}

Resveratrol (3,5,4' -trihydroxystilbene) is a phytoalexin and was first discovered in grapes in 1976. It contains 2 phenolic rings connected by a double bond and has 
2 isoforms: trans-resveratrol and cis-resveratrol, with the former being more stable [4]. Recently, it has been reported that resveratrol can inhibit inflammatory cytokine expression in response to lipopolysaccharide challenge in rat lungs [5]. Resveratrol can also inhibit the activation of transcription factors, such as NF- $\mathrm{BB}$ and AP-1 in monocytic U937 cells and alveolar epithelial A549 cells [6, 7]. Resveratrol can inhibit phorbol ester (PMA)-induced cyclooxygenase-2 (COX-2) activation, matrix metalloproteinases, adhesion molecules, and inducible nitric oxide synthase gene via down-modulation of NF- $\mathrm{kB}$ activation $[8,9]$. Furthermore, resveratrol inhibits the activation of c-Jun N-terminal kinases (JNK) [10] and its upstream kinase, mitogenactivated protein kinase [11]. Therefore, it appears that resveratrol can modulate a variety of pro-inflammatory pathways via inhibiting NF- $\kappa \mathrm{B}$ and MAPK activation.

\section{Curcumin}

Curcumin, a yellow-colored compound, is a member of the curcuminoid family of compounds obtained from the rhizome of Curcuma longa L. (family Zingiberaceae). Curcumin is one of the most extensively studied polyphenols and is reported to have a wide variety of effects, such as anti-inflammatory, antibacterial, antiviral, antifungal, antitumor, antispasmodic and hepatoprotective [12].

Curcumin inhibits NF- $\kappa \mathrm{B}$ activation, along with suppressing IL-8 release, COX-2 expression, and neutrophil recruitment in the lung [2]. Curcumin inhibits cigarette smoke-induced NF- $\kappa \mathrm{B}$ activation by inhibiting I $\kappa \mathrm{B} \alpha$ kinase in human lung epithelial cells [13]. Similar to resveratrol, curcumin also down-regulates various NF- $\kappa B-$ regulated genes that are involved in inflammation, such as leukotrienes, phospholipase A2, 5-lipoxygenase, adhesion molecules, inducible nitric oxide synthase and COX-2. In different cell types, various kinase signaling pathways, such as JNK, p38, AKT, JAK, ERK and PKC, are also modulated by curcumin [14]. Therefore, to identify the actual mechanism by which curcumin exerts its anti-inflammatory effects is complicated by its pleiotropic nature due to its ability to target so many different cellular signaling pathways. However, it may be possible that the ability of curcumin to prevent cross-talk between myriad signaling pathways might be a pre-requisite for its anti-inflammatory properties.

\section{Catechins}

These are monomeric flavanols comprising of chemically similar compounds, such as catechin, epicatechin, epigallocatechin, epicatechin gallate, and epigallocatechin gallate (EGCG) [15]. EGCG predominates among the various tea polyphenols and is considered to be the major therapeutic agent. EGCG has been shown to inhibit cigarette smoke extract-induced pro-inflammatory cytokine release in lung epithelial cells [16]. EGCG decreased NF- $\kappa B$ activity through hypoacetylation of RelA/p65 by directly inhibiting the activity of HAT [17]. EGCG has also been shown to modulate NF- $\kappa B / A P-1$ activity in PMA-stimulated mouse epidermal JB6 cells via inactivation of AP-1 [18] and/or NF- $\kappa B$ [19]. Similar to curcumin, green tea polyphenols also 
modulate myriad inflammatory signaling pathways [9] and therefore a single pathway cannot be assigned to the anti-inflammatory properties of these compounds.

\section{Deacetylases and Inflammation}

HDACs remove acetyl moieties from the $\varepsilon$-acetamido group on lysine residues of histones. The resulting deacetylation leads to chromatin condensation and therefore gene silencing. In addition to deacetylation of histones, HDACs can also deacetylate non-histone proteins, such as NF- $\kappa \mathrm{B}$, thus regulating NF- $\kappa \mathrm{B}$-dependent pro-inflammatory gene transcription [20]. Earlier investigations from our laboratory have shown that cigarette smoke-induced reduction in HDAC2 was concomitant to increased levels of total and acetylated RelA/p65 [20, 21]. Furthermore, the study revealed that RelA/p65 interacts with HDAC2 and RelA/p65 is retained in the nucleus, leading to activation of pro-inflammatory gene transcription when HDAC2 is deficient [20,21]. It is important to note that there is a significant decrement in the expression/activity of HDAC2 in lung parenchyma, bronchial biopsies and alveolar macrophages of COPD patients [22]. Decrease in the expression/activity of HDAC2 negatively correlated with the disease severity and the intensity of lung inflammation $[22,23]$. In contrast to increased HAT activity in bronchial biopsies and alveolar macrophages of asthmatics [24], there was no observed alteration in HAT activity in the lungs of COPD subjects [23].

Sirtuins (SIRT) belong to class III HDACs. They were the first to be reported to determine life span in yeast and the nematode. Unlike class I and II deacetylases, sirtuins are $\mathrm{NAD}^{+}$-dependent and are not inhibited by trichostatin $\mathrm{A}$ [25]. Since sirtuins require $\mathrm{NAD}^{+}$coming from metabolic reactions, it is hypothesized that sirtuins might act as a molecular link between cellular metabolic status (expressed by the $\mathrm{NAD}^{+}$/ $\mathrm{NADH}$ levels) and cellular transcription [26]. The best characterized and studied among the sirtuins is SIRT1, which is activated by polyphenolic compounds such as resveratrol. It is a nuclear deacetylase which primarily but not exclusively deacetylates proteins involved in transcriptional regulation. SIRT1 can therefore influence a wide range of physiological aspects, such as apoptosis/cell survival, autophagy, chromatin remodeling, gene transcription, senescence, endocrine signaling, and differentiation.

\section{HDAC2 and Steroid Resistance}

Chronic resistance to glucocorticoids is observed in patients with moderate to severe COPD and in asthmatics who smoke. HDAC2 is an important mediator of glucocorticoid activity and is found to be reduced in lungs of COPD patients and those of rodents exposed to oxidative stress or cigarette smoke. Molecular mechanisms, such as activation of NF- $\kappa \mathrm{B}$ and mitogen-activated protein kinase pathways, over-activation AP-1, reduced HDAC2 expression, and increased macrophage migration inhibitory factor have been implicated in onset of glucocorticoid resistance [27]. 
HDAC2 is required for the anti-inflammatory effects of glucocorticoids in COPD patients. Thus, reduction in the levels/activity of HDAC2 leads to corticosteroid resistance in such patients $[22,28]$. Polyphenolic compounds, such as theophylline have been shown to significantly increase HDAC2 activity thereby enhancing dexamethasone-induced suppression of IL-8 release in alveolar macrophage of COPD subjects [29] and exacerbations of COPD patients [30]. Furthermore, the ability of the HDAC2 to deacetylate glucocorticoid receptor (GR) enables GR to associate with RelA/p65, which leads to the attenuation of pro-inflammatory gene transcription [28]. Therefore, therapeutic restoration of HDAC2-dependent deacetylation of RelA/ p65 and GR appears to be a good strategy for enhancing glucocorticoid sensitivity.

\section{Sirtuins and Epigenetic Changes}

The epigenetic effects of SIRT1 can be appreciated in view of the ability of SIRT1 to deacetylate various transcription factors such as $\mathrm{p} 53$, forkhead transcription factor (FOXO), NF- $\kappa \mathrm{B}$, and histone proteins. Some of the physiological phenomena regulated by these transcription factors in response to environmental and toxic challenges include stress resistance, apoptosis, and inflammation [31]. While acetylation of FOXO3 leads to its inactivation, deacetylation by SIRT1 leads to its activation. Therefore, it can be surmised that SIRT1-mediated deacetylation of FOXO3 can induce cell cycle arrest, a phenomena altered in cancer cells. Furthermore, increased transcription of GADD45 (DNA repair system) and MnSOD (reactive oxygen detoxification) is a direct physiological consequence of deacetylation of FOXO3 by SIRT1 [32].

A series of reports have now emphasized the role of SIRT1 in epigenetic regulation of gene expression in cancer cells. Hyperacetylation of H4-K16 and decreased trimethylation of H3-K9 and H4-K20 have been observed after down-regulation of SIRT1 by siRNA in mammalian cells [33]. SIRT1 preferentially deacetylates H4-K16 in vitro [33]. In addition, loss of H4-K16 acetylation and H4-K20 trimethylation has been a hallmark in various tumors and tumor-derived cell lines, suggesting that these modifications may be characteristic epigenetic markers of cancer [34]. Promoter regions of tumor-suppressor genes whose DNA are hypermethylated and are silenced in many types of cancers are characteristic sites of localization of SIRT1 [35]. Such silenced genes were up-regulated in breast and colon cancer sells by down-regulation of SIRT1 levels/activity via increased H4-K16 as well as H3-K9 acetylation in such promoters [35]. Thus SIRT1-mediated epigenetic changes may play an important role in the modulation of various types of cancers and modulation of SIRT1 by polyphenols may serve as a chemopreventive agent.

\section{Modulation of Deacetylases by Dietary Polyphenols}

Dietary polyphenols, such as resveratrol, curcumin and catechins are shown to modulate NF- $\kappa$ B activation and chromatin remodeling through modulation of SIRT1 and 

Nutrigenetics
Nutrigenomics

Jutrigenet Nutrigenomics 2010;3:220-230

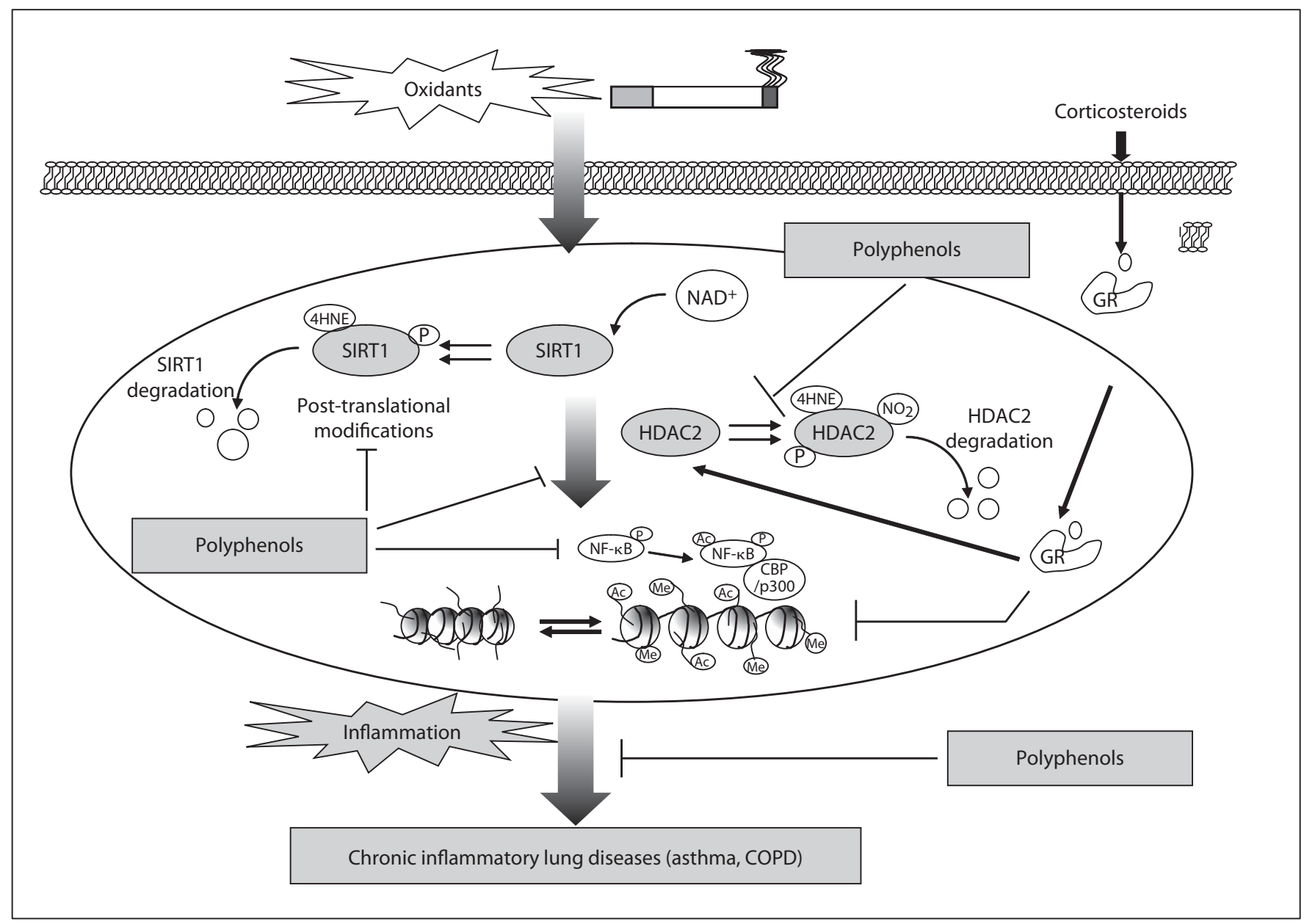

Fig. 2. Regulation of lung inflammation and chromatin remodeling by polyphenols. Dietary polyphenols modulate cigarette smoke and oxidants-mediated human lung inflammation by regulation of histone modifications. Cigarette smoke/oxidants inhibit histone deacetylases, such as SIRT1 and HDAC2 (by post-translational modifications), and/or trigger cellular signaling process leading to histone modifications. These epigenetic changes can cause abnormal activation or silencing of genes subsequent transcriptional repression or activation. Dietary polyphenols inhibit degradation of SIRT1 and HDAC2, and restore glucocorticoid efficacy, culminating in inhibition of chronic inflammatory response in the lung. $\mathrm{Ac}=$ acetylated, $\mathrm{p}=$ phosphorylated, $\mathrm{NO}_{2}=$ nitric oxide, $\mathrm{GR}=$ glucocorticoid receptor.

HDAC2 activity attenuating inflammatory gene expression in lung epithelium and macrophages (fig. 2). NF- $\kappa \mathrm{B}$ (due to intrinsic HAT activity) can lead to acetylation of histones thus causing epigenetic effects.

\section{Modulation of SIRT1}

A wide variety of compounds have now been identified, which can inhibit and/or activate sirtuins. Resveratrol, which activates SIRT1, deactivates p53 by significantly inhibiting p53 acetylation or by increased deacetylation of p53 [36] and protects from p53-mediated cellular apoptosis. In addition, resveratrol can also impart protection 
against Bax-induced apoptosis by favoring SIRT1-induced formation of Ku70-Bax complex [37]. Resveratrol has also been reported to increase DNA repair capacity and stress resistance by FOXO1/3-dependent expression of GADD43 and p27kip1 [38]. Such an effect has also been reported for other sirtuin-activating natural products, such as quercetin. Thus, resveratrol can impart cellular protection via modulating multiple targets.

Alternatively, cancer cells might be targeted using sirtuin inhibitors. These inhibitors induce cell damage by sensitizing the cells to p53-dependent apoptosis [36]. Pharmacological inhibition of SIRT1 decreases cellular resistance to stress and hence promotes cellular apoptosis due to reduced constraint on FOXO3/4 otherwise inhibited by SIRT1 [39]. SIRT1 is known to sensitize tumor cells to TNF- $\alpha$ induced cell death via inhibiting transactivation of NF- $\kappa B$ [40]. Thus it appears that SIRT1 inhibitors might yield cytoprotective effects by desensitizing the cells to TNF- $\alpha$ and therefore prevent cell death. Recently, it has been shown that SIRT1 activators also inhibit NF- $\mathrm{kB}$-mediated inflammatory mediators release and possibly overcome steroid-resistance in response to oxidative stress [41-43]. Therefore, it can be surmised that modulators of sirtuins might act as novel anti-inflammatory drugs via modulation of NF- $\kappa \mathrm{B}$. Furthermore, reports emerge it is becoming increasingly attractive to consider whether a combination of sirtuin inhibitors and DNA damaging anti-tumor drugs might offer a novel strategy for effective chemotherapeutic cancer therapy.

\section{Modulation of HDACs}

Inhibition of HDACs is a new concept in cancer chemoprevention. Of the many HDAC inhibitors known, butyrate, diallyl disulfide, and sulforaphane (SFN) are reported to exhibit anticancer properties [44]. However, in contrast to the traditional HDAC inhibitors, such as trichostatin A or SAHA, which are effective at lower concentrations (nanomolar range), the new range of dietary HDAC inhibitors are required in greater concentrations (micromolar range) [45]. Therefore, it is important to determine whether or not the concentrations of the new class of inhibitors are achieved under normal physiological conditions.

Of all the types of HDAC inhibitors known, butyrate is the smallest in size and can fully fit into the HDAC active site. The inhibitory effect of butyrate is exhibited in vitro between the high micromolar to low millimolar range, which might be achievable in the intestinal tract. The possibility of achievement of such a high concentration within the intestinal tract arises from the fact that colonocytes use butyrate as an oxidative fuel. Diallyl disulfide, found in garlic, is another HDAC inhibitor [46]. In vivo, it is metabolically converted to $S$-allylmercaptocysteine and its structure is similar to butyrate except that it has a 'spacer' ending with a carboxylate group [47]. SFN-cysteine contains a similar spacer and is a metabolite of SFN found in broccoli and broccoli sprouts. In the concentration range 3-15 mM, SFN-cysteine significantly inhibits HDAC activity [48]. In contrast, the parent compound SFN alone 
had no effect on HDAC activity. However, little is known about the distribution and concentrations of SFN and its active form(s) in different tissues. Although there are many dietary compounds that have HDAC inhibitory properties, more investigations are required in order to understand their bioavailability and the achievable concentrations of these compounds within the body.

Restoration of glucocorticoid function by curcumin is mediated through up-regulation of HDAC2 activity and restoration of HDAC1 and HDAC3 levels [20, 21]. Therefore, polyphenol-dependent redressing of HAT-HDAC imbalance has a significant impact on the epigenome and therefore inflammation, a concept that is corroborated by another report showing the ability of curcumin to inhibit HAT activity at very high concentration $(100 \mathrm{mM})$ and stalling NF- $\mathrm{KB}$-mediated histone acetylation [49]. Alternative mechanisms of polyphenol-mediated inhibition of inflammatory response could be through the reversing post-translational protein modifications of HDAC2 induced by oxidants and reactive aldehydes.

Corticosteroids have been one the major modes of therapy against various respiratory diseases, such as asthma and COPD. Failure of corticosteroids to ameliorate such disease conditions has been attributed to their failure to either recruit HDAC2 and SIRT1 or to the presence of an oxidatively modified HDAC2/SIRT1 in asthmatics and COPD patients. As discussed above, modulation of HDAC2/SIRT1 by dietary polyphenolic compounds may be useful in overcoming the steroid resistance in patients with asthma and COPD.

\section{Conclusions}

Epigenetics changes are increasingly believed to modulate the development and progression of many diseases including cancer and chronic respiratory disorders. It is important to understand whether or not a common target is shared by the class I \& II HDACs and sirtuins, so that a common therapeutic agent may be designed. Recent reports highlight the pharmacological significance of sirtuin-modulating drugs, and also suggest that identification of substrates specifically targeted by a single class of deacetylases, e.g. SIRT1 would have considerable therapeutic implications in chronic inflammatory diseases.

There are emerging reports that epigenetic alterations might be associated with chronic lung diseases. Recent advances in asthma and COPD research have suggested that epigenetic mechanisms, such as genomicimprinting, histone modification, DNA methylation of regulatory sequences other genes, and regulation by microRNA may also contribute to the susceptibility and complexity of the disease (including in utero) and hence dietary polyphenols may play a pivotal role in regulating these epigenetic modifications. The anti-inflammatory property of curcumin, resveratrol, and catechins may be due to their ability to induce HDAC2 activity and thereby restore the efficacy of glucocorticoids or overcome its resistance. Thus, regulation of inflammation 
Journal of
Nutrigenetics
and
Nutrigenomics
Jutrigenet Nutrigenomics 2010;3:220-230

DOI: 10.1159/000324358

Published online: April 6, 2011

(c) 2011 S. Karger AG, Basel

www.karger.com/jnn

by dietary polyphenols is potential therapeutic value against chronic inflammatory epigenetically-regulated diseases.

\section{Acknowledgments}

Supported by the NIH-NHLBI grants RO1-HL-085613, RO1-HL-097751-01, and NIEHS Environmental Health Sciences Center Grant ES-01247.

\section{References}

1 Aggarwal BB, Shishodia S: Suppression of the nuclear factor-kappaB activation pathway by spicederived phytochemicals: reasoning for seasoning. Ann NY Acad Sci 2004;1030:434-441.

-2 Biswas SK, McClure D, Jimenez LA, Megson IL, Rahman I: Curcumin induces glutathione biosynthesis and inhibits NF-kappaB activation and interleukin-8 release in alveolar epithelial cells: mechanism of free radical scavenging activity. Antioxid Redox Signal 2005;7:32-41.

- 3 Rahman I, Biswas SK, Kirkham PA: Regulation of inflammation and redox signaling by dietary polyphenols. Biochem Pharmacol 2006;72:1439-1452.

-4 Sovak M: Grape extract, resveratrol, and its analogs: a review. J Med Food 2001;4:93-105.

5 Birrell MA, McCluskie K, Wong S, et al: Resveratrol, an extract of red wine, inhibits lipopolysaccharide induced airway neutrophilia and inflammatory mediators through an NF-kappaB-independent mechanism. FASEB J 2005;19:840-841.

-6 Donnelly LE, Newton R, Kennedy GE, et al: Antiinflammatory effects of resveratrol in lung epithelial cells: molecular mechanisms. Am J Physiol Lung Cell Mol Physiol 2004;287:L774-L783.

-7 Manna SK, Mukhopadhyay A, Aggarwal BB: Resveratrol suppresses TNF-induced activation of nuclear transcription factors NF-kappa B activator protein-1, and apoptosis: potential role of reactive oxygen intermediates and lipid peroxidation. J Immunol 2000;164:6509-6519.

8 Leiro J, Arranz JA, Fraiz N, et al: Effect of cis-resveratrol on genes involved in nuclear factor kappa B signaling. Int Immunopharmacol 2005;5:393-406.

-9 Biesalski HK: Polyphenols and inflammation: basic interactions. Curr Opin Clin Nutr Metab Care 2007; 10:724-728.
10 Yang YT, Weng CT, Ho CT, Yen GC: Resveratrol analog-3,5,4'-trimethoxy-trans-stilbene inhibits invasion of human lung adenocarcinoma cells by suppressing the MAPK pathway and decreasing matrix metalloproteinase-2 expression. Mol Nutr Food Res 2009;53:407-416.

11 Venkatesan B, Ghosh-Choudhury N, Das F, et al: Resveratrol inhibits PDGF receptor mitogenic signaling in mesangial cells: role of PTP1B. FASEB J 2008;22:3469-3482.

12 Aggarwal BB, Kumar A, Bharti AC: Anticancer potential of curcumin: preclinical and clinical studies. Anticancer Res 2003;23:363-398.

13 Shishodia S, Potdar P, Gairola CG, Aggarwal BB: Curcumin (diferuloylmethane) down-regulates cigarette smoke-induced NF-kappaB activation through inhibition of IkappaBalpha kinase in human lung epithelial cells: correlation with suppression of COX-2 MMP-9 and cyclin D1. Carcinogenesis 2003;24:1269-1279.

14 Duvoix A, Blasius R, Delhalle $S$, et al: Chemopreventive and therapeutic effects of curcumin. Cancer Lett 2005;223:181-190.

15 Beecher GR: Overview of dietary flavonoids: nomenclature occurrence and intake. J Nutr 2003; 133:3248S-3254S.

6 Syed DN, Afaq F, Kweon MH, et al: Green tea polyphenol EGCG suppresses cigarette smoke condensate-induced NF-kappaB activation in normal human bronchial epithelial cells. Oncogene 2007; 26:673-682.

17 Choi KC, Jung MG, Lee YH, et al: Epigallocatechin3-gallate a histone acetyltransferase inhibitor inhibits EBV-induced B lymphocyte transformation via suppression of RelA acetylation. Cancer Res 2009;69:583-592.

18 Dong Z, Ma W, Huang C, Yang CS: Inhibition of tumor promoter-induced activator protein 1 activation and cell transformation by tea polyphenols, (-)-epigallocatechin gallate, and theaflavins. Cancer Res 1997;57:4414-4419. 


\section{Nutrigenetics Nutrigenomics}

\begin{tabular}{l|l}
\hline J Nutrigenet Nutrigenomics 2010;3:220-230 \\
\hline \begin{tabular}{l|l} 
DOI: 10.1159/000324358 \\
Published online: April 6, 2011
\end{tabular} & $\begin{array}{l}\text { @ } 2011 \text { S. Karger AG, Basel } \\
\text { www.karger.com/jnn }\end{array}$ \\
\hline
\end{tabular}

Rahman et al.: Polyphenols and Chromatin Remodeling in Inflammation
19 Nomura M, Ma W, Chen N, Bode AM, Dong Z: Inhibition of 12-O-tetradecanoylphorbol-13acetate-induced NF-kappaB activation by tea polyphenols (-)-epigallocatechin gallate and theaflavins. Carcinogenesis 2000;21:1885-1890.

-20 Yang SR, Chida AS, Bauter MR, et al: Cigarette smoke induces proinflammatory cytokine release by activation of NF-kappaB and posttranslational modifications of histone deacetylase in macrophages. Am J Physiol Lung Cell Mol Physiol 2006; 291:L46-L57.

-21 Meja KK, Rajendrasozhan S, Adenuga D, et al: Curcumin restores corticosteroid function in monocytes exposed to oxidants by maintaining HDAC2. Am J Respir Cell Mol Biol 2008;39:312-323.

22 Ito K, Ito M, Elliott WM, et al: Decreased histon deacetylase activity in chronic obstructive pulmonary disease. N Engl J Med 2005;352:1967-1976.

-23 Adenuga D, Yao H, March TH, Seagrave J, Rahman I: Histone deacetylase 2 is phosphorylated, ubiquit- 38 inated, and degraded by cigarette smoke. Am J Respir Cell Mol Biol 2009;40:464-473.

24 Barnes PJ, Adcock IM, Ito K: Histone acetylation and deacetylation: importance in inflammatory 39 lung diseases. Eur Respir J 2005;25:552-563.

-25 Imai S, Armstrong CM, Kaeberlein M, Guarente L: Transcriptional silencing and longevity protein Sir2 is an NAD-dependent histone deacetylase. Nature 2000;403:795-800.

-26 Bordone L, Guarente L: Calorie restriction, SIRT1 and metabolism: understanding longevity. Nat Rev Mol Cell Biol 2005;6:298-305.

-27 Barnes PJ, Adcock IM: Glucocorticoid resistance in inflammatory diseases. Lancet 2009;373:19051917.

28 Ito K, Yamamura S, Essilfie-Quaye S, et al: Histone deacetylase 2-mediated deacetylation of the glucocorticoid receptor enables NF-kappaB suppression J Exp Med 2006;203:7-13.

29 Cosio BG, Tsaprouni L, Ito K, et al: Theophylline restores histone deacetylase activity and steroid responses in COPD macrophages. J Exp Med 2004; 200:689-695.

30 Cosio BG, Iglesias A, Rios A, et al: Low-dose theophylline enhances the anti-inflammatory effects of steroids during exacerbations of COPD. Thorax 2009;64:424-429.

-31 Yang T, Sauve AA: NAD metabolism and sirtuins: metabolic regulation of protein deacetylation in stress and toxicity. AAPS J 2006;8:E632-E643.

- 32 Kobayashi Y, Furukawa-Hibi Y, Chen C, et al: SIRT1 is critical regulator of FOXO-mediated transcription in response to oxidative stress. Int J Mol Med 2005; 16:237-243.
Vaquero A, Scher M, Lee D, et al: Human SirT1 interacts with histone $\mathrm{H} 1$ and promotes formation of facultative heterochromatin. Mol Cell 2004;16:93105.

34 Fraga MF, Ballestar E, Villar-Garea A, et al: Loss of acetylation at Lys 16 and trimethylation at Lys20 of histone $\mathrm{H} 4$ is a common hallmark of human cancer. Nat Genet 2005;37:391-400.

35 Pruitt K, Zinn RL, Ohm JE, et al: Inhibition of SIRT1 reactivates silenced cancer genes without loss of promoter DNA hypermethylation. PLoS Genet 2006;2:e40

36 Howitz KT, Bitterman KJ, Cohen HY, et al: Small molecule activators of sirtuins extend Saccharomyces cerevisiae lifespan. Nature 2003;425:191-196.

7 Cohen HY, Miller C, Bitterman KJ, et al: Calorie restriction promotes mammalian cell survival by inducing the SIRT1 deacetylase. Science 2004;305: 390-392.

8 Daitoku H, Hatta M, Matsuzaki H, et al: Silent information regulator 2 potentiates Foxo1-mediated transcription through its deacetylase activity. Proc Natl Acad Sci USA 2004;101:10042-10047.

Brunet A, Sweeney LB, Sturgill JF, et al: Stressdependent regulation of FOXO transcription factors by the SIRT1 deacetylase. Science 2004;303:20112015.

40 Yeung F, Hoberg JE, Ramsey CS, et al: Modulation of NF-kappaB-dependent transcription and cell survival by the SIRT1 deacetylase. Embo J 2004;23: 2369-2380.

41 Nakamaru Y, Vuppusetty C, Wada H, et al: A protein deacetylase SIRT1 is a negative regulator of metalloproteinase-9. FASEB J 2009;23:2810-2819.

- 42 Rajendrasozhan S, Yang SR, Kinnula VL, Rahman I: SIRT1 an antiinflammatory and antiaging protein is decreased in lungs of patients with chronic obstructive pulmonary disease. Am J Respir Crit Care Med 2008;177:861-870.

43 Yang SR, Wright J, Bauter M, et al: Sirtuin regulates cigarette smoke-induced proinflammatory mediator release via RelA/p65 NF-kappaB in macrophages in vitro and in rat lungs in vivo: implications for chronic inflammation and aging. Am J Physiol Lung Cell Mol Physiol 2007;292:L567-L576.

44 Myzak MC, Dashwood RH: Histone deacetylases as targets for dietary cancer preventive agents: lessons learned with butyrate diallyl disulfide and sulforaphane. Curr Drug Targets 2006;7:443-452.

45 Dashwood RH, Myzak MC, Ho E: Dietary HDAC inhibitors: time to rethink weak ligands in cancer chemoprevention? Carcinogenesis 2006;27:344349 . 
46 Druesne N, Pagniez A, Mayeur C, et al: Diallyl disulfide (DADS) increases histone acetylation and p21(waf1/cip1) expression in human colon tumor cell lines Carcinogenesis 2004;25:1227-1236.

$\checkmark 47$ Guyonnet D, Bergès R, Siess $\mathrm{MH}$, et al: Postinitiation modulating effects of allyl sulfides in rat hepatocarcinogenesis. Food Chem Toxicol 2004;42: 1479-1485.
-48 Myzak MC, Karplus PA, Chung FL, Dashwood RH: A novel mechanism of chemoprotection by sulforaphane: inhibition of histone deacetylase. Cancer Res 2004;64:5767-5774.

49 Kang J, Chen J, Shi Y, Jia J, Zhang Y: Curcumininduced histone hypoacetylation: the role of reactive oxygen species. Biochem Pharmacol 2005;69: 1205-1213. 